\title{
SZ Lyn: New BVRI CCD observations and improved pulsational and orbital elements
}

\author{
K.D. Gazeas, P.G. Niarchos, K.A. Boutsia \\ Department of Astrophysics, Astronomy and Mechanics, National and Kapodistrian \\ University of Athens, GR 15784 Zographou, Athens, Greece
}

\begin{abstract}
New BVRI CCD observations of the pulsating star SZ Lyn are presented. The light curves are analyzed using Fourier transform techniques and a new period is determined. A new ephemeris, based on recent times of maxima, is proposed. The photometric data collected over the last 40 years are used to determine the pulsational and orbital elements, since SZ Lyncis is a member of a binary system. The results are compared with those found in previous studies.
\end{abstract}

\section{Introduction}

SZ Lyncis $\left(=\mathrm{BD}+44^{\circ} 1718,=\mathrm{HD} 67390\right)$ is a high-amplitude $\delta$ Scuti star, discovered by Hoffmeister in 1949. The pulsational behaviour was determined for the first time by Schneller (1961). Apart from small ( $<0.02 \mathrm{mag})$ cycle-to-cycle variations, no irregularities have been found in the star's light variations and all attempts to find double mode pulsation have failed. Van Genderen (1967) noticed that the residuals in the $\mathrm{O}-\mathrm{C}$ diagrams appeared to follow a sinusoidal variation with a period of $\mathrm{P}=3.091 \pm 0.051$ years. Several years later, Barnes \& Moffett (1975) improved this period to $P=3.138 \pm 0.028$ years and suggested that the sinusoidal variations in the $\mathrm{O}-\mathrm{C}$ diagrams, observed by van Genderen (1967), are light travel time effect, since SZ Lyn is a member of a binary system. This hypothesis was confirmed by Bardin \& Imbert (1984) with measurements of radial velocities. Their results fully confirmed the binary nature of the system and led to an orbit with a fairly large computed eccentricity. The ephemeris given by Binnendijk (1968) is: 
$\mathrm{t}_{\max }(\mathrm{HJD})=2439121.7003+0^{d} .12053188 \times \mathrm{E}$

Soliman et al. (1986) found the pulsation period to be 0.120534896 days and the orbital period $1173.5 \pm 2.0$ days. From the BVRI observations during the period January 1975 - March 1979, Moffett et al. (1988) estimated the pulsational period as 0.12052115 days, which is shorter than earlier determinations. The latest research was made by Paparó et al. (1988), who improved the pulsational and orbital elements of the system, using all available data from 1961 until 1988. They proposed the following ephemeris:

$\mathrm{t}_{\max }(\mathrm{HJD})=2438124.39955(39)+0^{d} .120534910(13) \times \mathrm{E}$

\section{Observations and data reduction}

The new observations were made at the University of Athens Observatory, with a $40 \mathrm{~cm} \mathrm{f} / 8$ Cassegrain telescope. The observations were collected during 8 nights from December 2001 until May 2003. The star was observed in most of the nights for at least 3 hours, covering more than one period in Bessell $B, V$, $\mathrm{R}$ and I filters. Nine maxima were covered, 4 of which are in $\mathrm{V}$ filter, 2 in $\mathrm{R}$, 2 in $B$ filter and 1 in I filter. The photometric reduction was done using the software package AIP4WIN (Berry \& Burnell 2000). In total we used of 851 data points in our analysis in $B, V, R$, I filters.

The light curves from almost every observing night cover one minimum and one maximum. GSC 2979:1343 was used as the comparison star, while GSC 2979:1329, GSC 2979:1174 and GSC 2979:1370 were taken as check stars. They showed no sign of variability and the standard deviation of the points was typically about $20 \mathrm{mmag}$. In order to calculate the period we fitted a Gaussian curve to the data around the maxima. Using these values we derived the following linear ephemeris:

$\mathrm{t}_{\max }(\mathrm{HJD})=2452776.289(10)+0^{d} .1205349(41) \times \mathrm{E}$

\section{Light curve analysis and period search}

For the sake of homogeneity, the new times of maxima and the $\mathrm{O}-\mathrm{C}$ values in Table 1 were computed according to ephemeris given by equation 2 . 


\begin{tabular}{cccc}
\hline HJD of maximum & E (cycles) & O-C (days) & Rem. \\
\hline 2452263.6389 & 117304 & 0.0123 & $\mathrm{Pp}$ \\
2452374.4127 & 118223 & 0.0145 & $\mathrm{Pp}$ \\
2452410.3327 & 118521 & 0.0151 & $\mathrm{Pp}$ \\
2452774.3595 & 121541 & 0.0265 & $\mathrm{Pp}$ \\
2452775.3237 & 121549 & 0.0264 & $\mathrm{Pp}$ \\
2452776.2882 & 121557 & 0.0266 & $\mathrm{Pp}$ \\
\hline
\end{tabular}

$\mathrm{Pp}=$ Present paper.

Table 1: Times of maxima and $\mathrm{O}-\mathrm{C}$ values calculated according to the ephemeris (2).
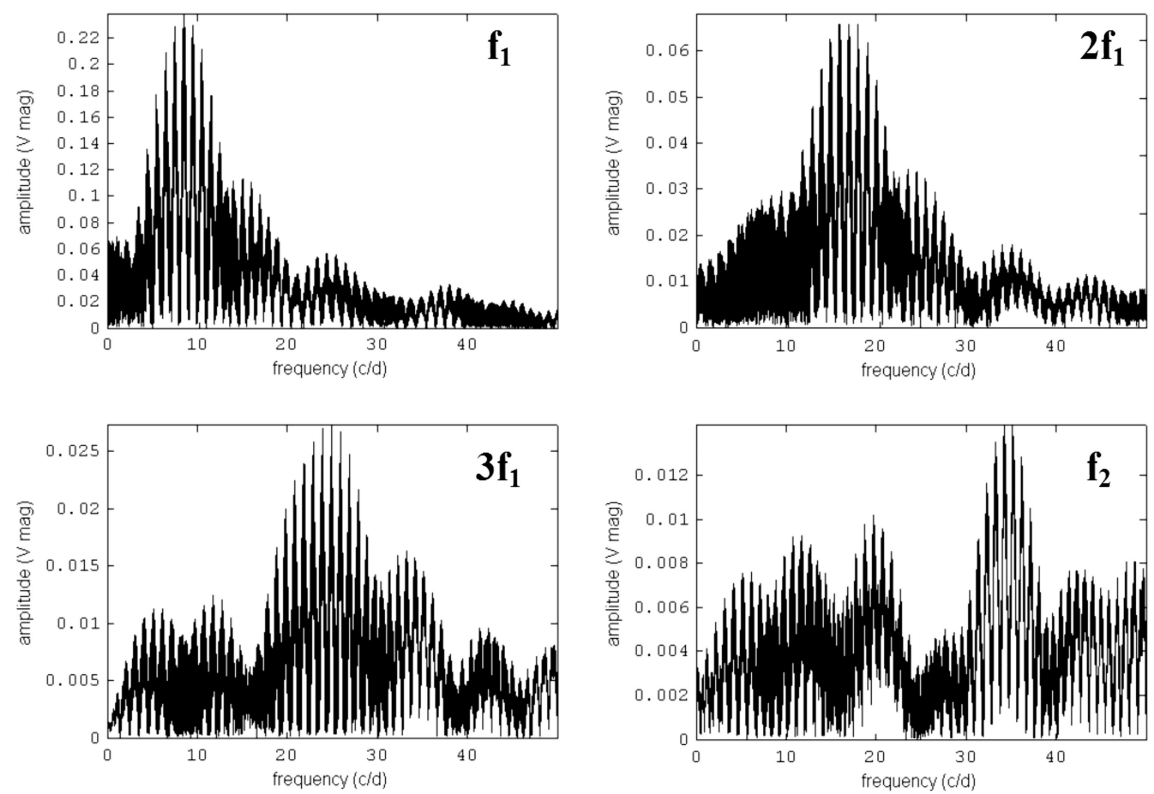

Figure 1: The amplitude spectra derived from the period searching.

During the last five nights (April 7, May 5, 13, 14 and 15, 2003) we have used only the $\mathrm{V}$ filter. The pulsating period was derived with a Fourier transformation, using the Period98 software (Sperl 1998). In the power spectrum we can see the main frequency for the pulsating star, which is 8.2965 (6) cycles/day and corresponds to a period of $0.1205332(87)$ days. After excluding the dom- 


\begin{tabular}{ccc}
\hline & Frequency (c/d) & Amplitude (mag) \\
\hline $\mathrm{f} 1$ & $8.2965(6)$ & $0.222(1)$ \\
$2 \mathrm{f} 1$ & $16.5930(6)$ & $0.073(1)$ \\
3f1 & $24.8887(6)$ & $0.029(1)$ \\
$\mathrm{f} 2$ & $34.2877(6)$ & $0.015(1)$ \\
\hline
\end{tabular}

Table 2: Frequencies found using the Fourier transform techniques.

inant frequency (prewhitening procedure), we searched for possible secondary frequencies. We found that the only frequencies, appearing after $f_{1}$, are its harmonics, $2 f_{1}$ and $3 f_{1}$ (Table 2). There is a possible existence of the harmonic $4 f_{1}$, but the very low amplitude and strong alias of other low power frequencies affect its value (frequency marked as $f_{2}$ in Table 2 ). In any case, the amplitude of this frequency is too low to be assumed as one of the apparent frequencies. The amplitude spectra are shown in Figure 1. The lack of other frequencies is probably due to the limited number of data. The search for a secondary frequency failed in the past too (Szeidl, 1983).

\section{Phased light curves}

We used our linear ephemeris (equation 3), derived from all our observations (214, 353, 242, 42 points in B, V, R and I filter, respectively), to phase the light curves from December 2001 to May 2003. The phase diagrams, as well as the color $(\mathrm{B}-\mathrm{V})$ curve, are presented in Figure 2. It can be seen that the minimum occurs around phase 0.7 and the amplitude of pulsation decreases as we go to longer wavelengths.

\section{O-C diagrams}

It is assumed that the $\mathrm{O}-\mathrm{C}$ variations are caused by the linear change of the pulsation period of SZ Lyn (A) and by the light travel time effect of SZ Lyn, orbiting in an elliptical orbit (B). We have used equation (2) to calculate the $\mathrm{O}-\mathrm{C}$ values for the times of maxima (presented in Table 2). It was obvious that the period continues to decrease. Paparó et al. (1988) found that the linear change of the pulsational period of SZ Lyn is $\beta=2.25 \pm 0.42 \times 10^{-12}$ days/cycle. This value could not fit our data very well. A new analysis of all available 


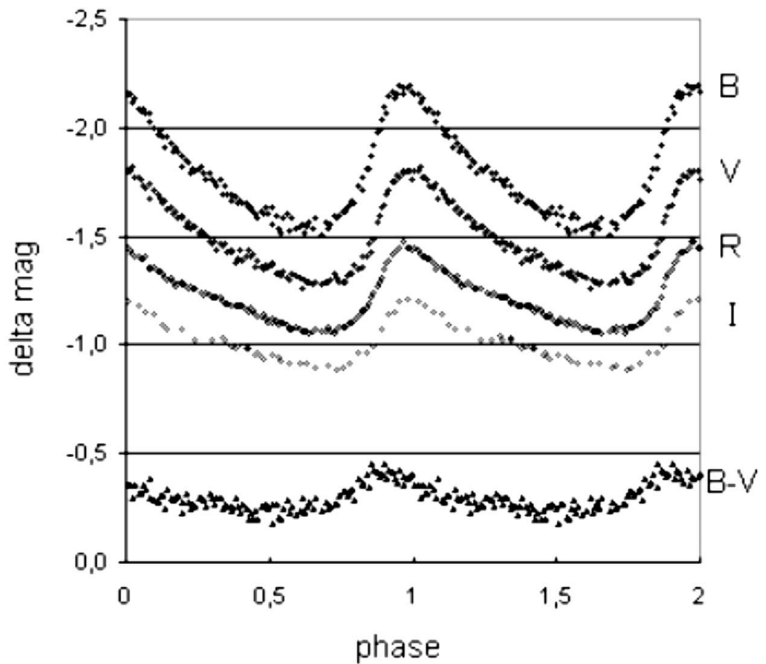

Figure 2: The new $B, V, R, I$ and $B-V$ phase diagrams.

times of maxima (from March 1961 until May 2003) yielded a value of $\beta=2.90$ $\pm 0.22 \times 10^{-12}$ days/cycle. The new $\mathrm{O}-\mathrm{C}$ diagram is presented in Figure 3 . The Gauss-Newton method was used to derive the orbital parameters of SZ Lyn. The results of a least squares fit are given in the first column of Table 3, together with the pulsational elements $\mathrm{T}_{0}$ and $\mathrm{P}_{0}$, derived from Equation 3 . For comparison, the second and third columns give the results of Paparó et al. (1988) derived photometricaly and spectroscopicaly, respectively. Figure 4 shows the residuals from the ephemeris $\mathrm{O}-\mathrm{C}_{A}$ versus phase in the 1179.3-day cycle, after the subtaction of the paraboloidal increase of the $\mathrm{O}-\mathrm{C}$ value (linear change).

\section{SZ Lyn as a member of a binary system}

The orbital period of the binary system is very close to 1180 days, $\left(\mathrm{P}_{\text {orb }}=\right.$ $1179.3 \pm 2$ days), similar to the value found in earlier studies. This period leads to the result that the two components are separated by about $1 \mathrm{AU}$ from each other. The pulsating star is the brighter component and it has a spectral type that ranges from A7-F2 (Kholopov et al. 1985). The faint component could not be observed so far and thus SZ Lyn is characterized as a single line spectroscopic binary. The linear change of the pulsational period of SZ Lyn 


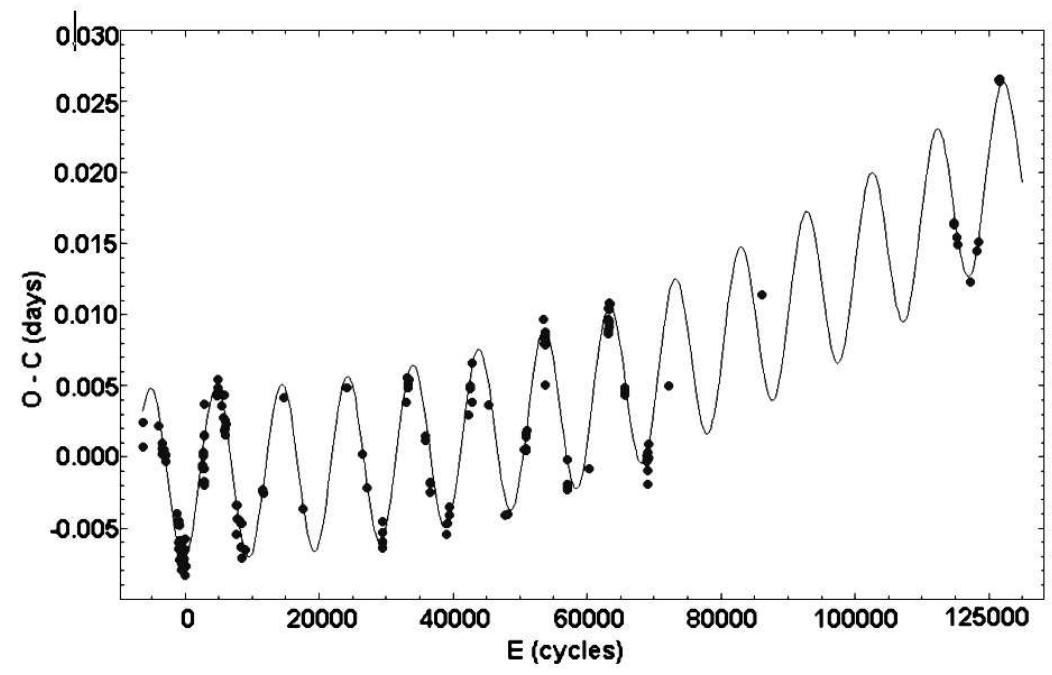

Figure 3: The O-C diagram of SZ Lyn, based on Equation 2.

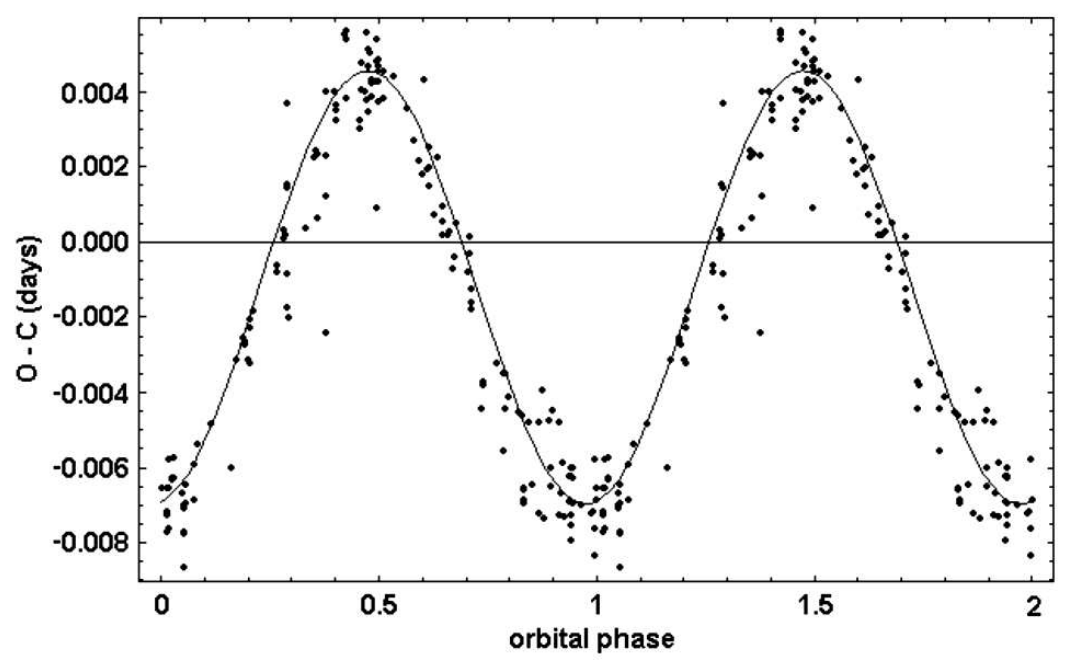

Figure 4: The residuals from the ephemeris $\mathrm{O}-\mathrm{C}_{A}$ versus phase in the 1179.3-day cycle. The smooth curve is the fit of the elliptic orbit. 
Table 3: Pulsational and orbital elements of SZ Lyn.

\begin{tabular}{lccc}
\hline & $\begin{array}{c}\text { From photometry } \\
\text { Present paper }\end{array}$ & $\begin{array}{c}\text { From photometry } \\
\text { Paparó et al. (1988) }\end{array}$ & $\begin{array}{c}\text { From spectroscopy } \\
\text { Paparó et al. (1988) }\end{array}$ \\
\hline $\mathrm{T}_{0}$ (HJD) & $2452776.289(10)$ & $2438124.39955(39)$ & - \\
$\mathrm{P}_{0}$ (days) & $0.1205349(41)$ & $0.120534910(13)$ & - \\
$\beta(\mathrm{c} / \mathrm{d})$ & $(2.90 \pm 0.22) \times 10^{-12}$ & $(2.25 \pm 0.42) \times 10^{-12}$ & - \\
$\mathrm{P}_{\text {orb }}$ (days) & $1179.3 \pm 2$ & $1177.7 \pm 2$ & $1177.7 \pm 2$ \\
$\mathrm{~A}($ days $)$ & $0.00599 \pm 0.00023$ & $0.00575 \pm 0.00013$ & - \\
$\mathrm{V}_{0}\left(\mathrm{kms}^{-1}\right)$ & - & - & $34.15 \pm 0.05$ \\
$\mathrm{~K}\left(\mathrm{kms}^{-1}\right)$ & - & - & $9.58 \pm 0.09$ \\
asini $(\mathrm{AU})$ & $0.998 \pm 0.04$ & $0.996 \pm 0.022$ & $1.017 \pm 0.013$ \\
$\mathrm{e}$ & $0.205 \pm 0.010$ & $0.200 \pm 0.047$ & $0.194 \pm 0.008$ \\
$\omega($ deg $)$ & $87.6 \pm 1.1$ & $123.3 \pm 14.1$ & $99.5 \pm 2.6$ \\
$\mathrm{~T}(\mathrm{HJD})$ & $2445699.8436 \pm 0.022$ & $2445857.6 \pm 47.7$ & $2445798.6 \pm 7.6$ \\
$\mathrm{f}(\mathrm{M})$ & $0.095 \pm 0.008$ & $0.095 \pm 0.007$ & $0.101 \pm 0.004$ \\
\hline
\end{tabular}

is found to be $\beta=2.90 \pm 0.22 \times 10^{-12}$ days/cycle. According to Fernley et al. (1984), the mass of SZ Lyn is estimated to be $\mathrm{M}_{S Z}=1.6 \mathrm{M}_{\odot}$, while the mass of the secondary component is estimated to be $0.8 \mathrm{M}_{\odot}<\mathrm{M}_{2}<1.2 \mathrm{M}_{\odot}$ (Szeidl 1983 \& Paparó et al. 1988). Later computations by McNamara (1997) showed that $\mathrm{M}_{S Z}=1.92 \mathrm{M}_{\odot}$ and $\mathrm{R}_{S Z}=3.18 \mathrm{R}_{\odot}$.

\section{Summary and conclusions}

Our new BVRI CCD photometric observations and those obtained by Derekas et al. (2003) and Hipparcos (ESA 1997), extended the time base of data from 27 to 42 years (or from 8 to 14 revolutions of the system). 165 times of maxima were observed between 1961 and 2003 and have been used to calculate the pulsational and orbital elements of the binary system. From the analysis we were able to calculate a more precise value of the linear change in the star's pulsational period. New improved values were also calculated for all the orbital parameters of the binary system. The analysis shows that the $\mathrm{O}-\mathrm{C}$ residuals are a powerful tool for investigating the binary nature of pulsating stars and 
deriving their orbital parameters.

Acknowledgments. Many thanks are due to B. Szeidl and M. Paparó for helpful discussions and comments. It is also a pleasure to thank E. Fragkoulopoulos for his help with the numerical computations of the present work.

\section{References}

Africano, J. 1978, IBVS, 1408

Bardin, C., Imbert, M. 1984, A\&AS, 57, 249

Barnes, T. G. III, Moffett, T. J. 1975, AJ, 80, 48

Berry, R., Burnell, J. 2001, The Handbook of Astronomical Image Processing

Software for Windows - Manual for AIP4WIN, Richmond

Binnendijk, L. 1968, AJ, 73, 29

Braune, W., Mundry, E. 1982, BAV Mitt., No. 34

Braune, W., Huebscher, J., Mundry, E. 1983, BAV Mitt., No. 36

Broglia, P. 1963, Mem. Soc. Astron. Ital., 34, 431

Derekas, A, Kiss, L.L., Szekely, P., Alfaro, E.J., Csak, B., Meszaros, Sz., Rodriguez,

E., Rolland, A., Sarneczky, K., Szabo, Gy.M., Szatmary, K., Varadi, M., Kiss, Cs. 2003, A\&A, 402, 733

Duerbeck, H.W. 1976, IBVS, 1171

Eggen, O. J. 1962, PASP, 74, 159

ESA 1997, The Hipparcos and Tycho Catalogues, ESA SP-1200

Fernley, J.A., Jameson, R.F., Sherrington, M.R. 1984, MNRAS, 208, 853

Garbusov, G. A. 1980, IBVS, 1854

Garrido, R., Alfaro, E. J., Quintana, J. M., Saez M. 1979, A\&AS, 36, 51

He Tian-jian, Xiong Da-run 1964, Acta Astron. Sinica, 12, 57

Hoffmeister, C. 1949, Astron. Abhand, 12, 1

Hopp, U., Witzigmann, S. 1979, IBVS, 1579

Jiang, S.-Y., Huang, Y.-W., Guo, Z.-H. 1985, Acta Astron. Sinica, 26, 135

Joshi, S.C., Srivastava, H.N. 1967, Z. Astrophys., 67, 456

Karetnikov, V. G., Medvedev, Y. A. 1977, IBVS, 1309

Kholopov, P.N. et al. 1985, General Catalogue of Variable Stars,

Fourth Edition, Moscow

McNamara, D.H. 1997, PASP, 109, 1221

Moffett, T.J., Barnes, T.G. III, Fekel, F.C. Jr., Jefferys, W. H., Achtermann Jeffrey, M. 1988, AJ, 95

Paparó, M., Szeidl, B., Mahdy Hamid, A. 1988, Ap\&SS, 149

Popovici, C. 1971, IBVS, 508 
Schneller, H. 1961, Astron. Nachr., 286, 102

Soliman, M.A., Hamdy, M.A., Szeidl, B., Szabados, L. 1986, Comm. Konkoly Obs.

Hung. Acad. Sci., Budapest, No 88 (Vol. 10, part 2)

Sperl, M. 1998, Manual for Period98 Software

Szeidl, B. 1983, Comm. Konkoly Obs. Hung. Acad. Sci., Budapest, No 84 (Vol. 9)

van Genderen, A.M. 1963, Bull. Astron. Inst. Neth., 17, 243

van Genderen, A.M. 1967, Bull. Astron. Inst. Neth., 19, 74

Wisse, M., Wisse, P.N.J. 1969, Bull. Astron. Inst. Neth., 20, 333 